Port Acadie

Revue interdisciplinaire en études acadiennes

An Interdisciplinary Review in Acadian Studies

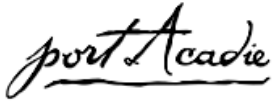

\title{
La politique patrimoniale de la Nouvelle-Écosse
}

\section{Jill Scott}

Numéro 10-11-12, automne 2006, printemps-automne 2007

Le patrimoine religieux de la Nouvelle-Écosse : signes et paradoxes en Acadie

URI : https://id.erudit.org/iderudit/018642ar

DOI : https://doi.org/10.7202/018642ar

Aller au sommaire du numéro

Éditeur(s)

Université Sainte-Anne

ISSN

1498-7651 (imprimé)

1916-7334 (numérique)

Découvrir la revue

Citer cet article

Scott, J. (2006). La politique patrimoniale de la Nouvelle-Écosse. Port Acadie, (10-11-12), 231-237. https://doi.org/10.7202/018642ar

\section{Résumé de l'article}

En 1980, la Nouvelle-Écosse adoptait sa première loi sur la protection du patrimoine bâti. Depuis, plus de 260 édifices ont reçu la désignation d'édifices historiques et sont, à cette fin, protégés en vertu de la loi. En 2003, la province a lancé sa participation officielle à l'Initiative des endroits historiques, qui a pour mandat de promouvoir et de reconnaître les endroits historiques désignés par la province et par les municipalités. Cet exposé présente le programme de protection des lieux historiques de la Nouvelle-Écosse et aborde la manière dont la province a contribué à la mise en place de l'Initiative des endroits historiques, qui est une initiative pancanadienne. 
TROISIÈME PARTIE : L'AVENIR DU PATRIMOINE RELIGIEUX

QUAND L'ÉTAT S'ENGAGE

\section{La politique patrimoniale de la Nouvelle-Écosse}

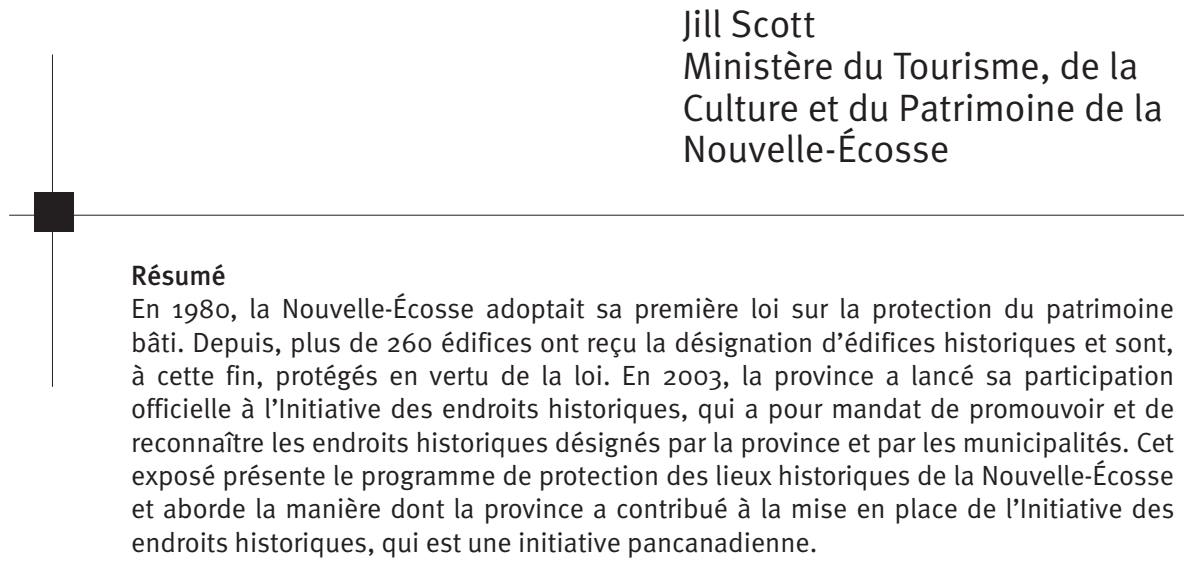

Un climat favorable

Suite à l'adoption de la Charte de Venise en 1965, qui définit les principes de la préservation du patrimoine, et de la Convention $d u$ patrimoine mondial de 1972, par laquelle les pays participants se sont engagés à protéger le patrimoine culturel et naturel, le mouvement de protection patrimoniale a pris de plus en plus d'ampleur dans le monde. Il a conduit à plusieurs mesures importantes au Canada en vue de mettre en place un climat favorable à la préservation du patrimoine. Les principes établis par la communauté internationale ont été adoptés et adaptés par des groupes de professionnels du secteur de la protection du patrimoine (architectes, urbanistes, archéologues, etc.), afin de se donner l'assise théorique dont on avait besoin dans le contexte canadien. La mise en place d'outils et de mécanismes destinés à favoriser la préservation du patrimoine a bénéficié du soutien d'instances importantes, tant au niveau gouvernemental que dans le secteur des organisations non gouvernementales. D'autres ont mis en pratique et peaufiné les techniques et les méthodes de préservation dans le cadre de leur travail au quotidien. L'impact de l'entrée en vigueur de ces mesures a permis de sensibiliser non seulement le grand public, mais également les gouvernements à la nécessité d'adopter de nouveaux textes de loi en matière de protection 
du patrimoine. Les gouvernements des provinces et des territoires du Canada, y compris la Nouvelle-Écosse, ont ainsi été amenés à créer leurs propres textes de loi en la matière. Le présent article décrit les textes de loi importants qui favorisent la protection du patrimoine néo-écossais, à savoir la loi sur les biens patrimoniaux (Heritage Property Act), la loi sur les endroits spéciaux (Special Places Act) et la loi sur la protection des cimetières (Cemeteries Protection Act).

\section{Les lois de la Nouvelle-Écosse}

\section{La loi sur les biens patrimoniaux}

La loi sur les biens patrimoniaux (dite Heritage Property Act), adoptée en 1980, concerne le repérage, la désignation et la protection des édifices, des structures, des régions et des arrondissements et vise à encourager la poursuite de l'utilisation de ces ressources culturelles. À cette fin, elle attribue divers pouvoirs, dont certains à la province et d'autres aux administrations municipales. La province a le pouvoir d'établir un "Registre des biens patrimoniaux », qui dresse la liste des lieux historiques en Nouvelle-Écosse qui sont considérés comme ayant de l'importance dans le patrimoine de la province. La loi donne aux municipalités le pouvoir d'établir un registre des biens patrimoniaux qui ont de l'importance dans le patrimoine au niveau local ou régional et le pouvoir d'établir des « arrondissements de préservation du patrimoine » - ce que le gouvernement provincial ne peut pas faire. La province et les municipalités peuvent aussi, si elles le souhaitent, apposer, en vertu de cette loi, des plaques sur les biens patrimoniaux inscrits au registre et protégés par la loi.

Le Programme des biens patrimoniaux (PBP) a été mis en place en 1980 en vue de remplir les obligations de la loi et relève de la Division du patrimoine du ministère du Tourisme, de la Culture et du Patrimoine de la Nouvelle-Écosse. Le PBP définit les exigences procédurales, fournit les désignations provinciales et municipales, attribue un petit montant d'aide financière, s'assure que les propriétaires de biens patrimoniaux disposent du soutien et des conseils dont ils ont besoin et tient à jour le Registre des biens patrimoniaux provinciaux de la Nouvelle-Écosse. En ce qui a trait à la condition des biens patrimoniaux, le ministre responsable du PBP bénéficie des avis du Conseil consultatif sur le patrimoine de la NouvelleÉcosse, qui compte entre cinq et douze membres. Conformément à la définition de la loi, le rôle de ce conseil est, entre autres responsabilités, d'examiner, au nom du ministre, les demandes concernant la désignation de nouveaux biens patrimoniaux, les demandes concernant des 
modifications substantielles à apporter aux biens patrimoniaux et les demandes de permis de démolition. En dernière analyse, cependant, c'est le ministre responsable de la loi sur les biens patrimoniaux qui décide de l'inscription des biens patrimoniaux au registre provincial.

Le processus de désignation des biens patrimoniaux provinciaux commence par une demande déposée par le propriétaire auprès du PBP. Il est à noter que tous les types de structures sont susceptibles de faire l'objet d'une demande, y compris les cimetières, les édifices industriels, commerciaux ou agricoles et même les parcs ou les structures faisant partie des parcs. Les structures relevant du patrimoine ne se limitent pas non plus à celles qui ont été bâties avant 1914. Il peut même s'agir d'un édifice construit au cours des quarante dernières années. Le personnel du PBP organise une visite de la propriété et entreprend les recherches exigées par le conseil consultatif. Les recherches faites au nom du conseil consultatif s'orientent selon des critères de sélection d'ordre général permettant de garantir que la loi soit appliquée de façon cohérente. Ces critères prennent en compte l'histoire et la culture, l'architecture et le contexte ou le caractère unique et la condition de ce qui est proposé. Les résultats des recherches sont ensuite distribués aux membres du conseil consultatif, à qui on présente également des diapositives de l'endroit, lors d'une réunion se déroulant par la suite. Ce sont les membres du conseil qui décident si la proposition respecte les critères établis pour l'inscription au registre provincial; les recommandations concernant l'inscription de biens patrimoniaux au registre sont transmises au ministre par l'intermédiaire du PBP. Le ministre transmet un avis de recommandation au propriétaire, dans lequel il indique les raisons justifiant l'inscription au Registre des biens patrimoniaux, les implications de cette inscription et les frontières de la propriété. Le propriétaire peut s'opposer à l'inscription au registre, du moment qu'il le fait dans les trente jours qui suivent l'avis de recommandation. On fournit également une copie de l'avis de recommandation au Bureau d'enregistrement des titres de propriété. Si personne ne présente d'objection, le ministre dispose d'une période supplémentaire de quatre-vingt-dix jours pour décider d'ajouterla propriété proposée au registre provincial. Une fois encore, lorsque la propriété est ajoutée au registre, le ministre envoie un avis de recommandation au propriétaire indiquant que la propriété fait désormais partie du Registre des biens patrimoniaux de la province. Ce document est également porté au registre des titres de propriété.

Dans le cas des municipalités, comme, par exemple, la ville de Yarmouth ou la ville de Wolfville, conformément à l'arrêté municipal en matière de patrimoine, le conseil municipal a le pouvoir d'établir un registre municipal des biens patrimoniaux. C'est un comité consultatif 
de la municipalité qui se charge de recommander l'ajout de propriétés au registre municipal. Les membres du comité sont nommés par le conseil municipal et incluent au moins deux membres du conseil municipal. Chaque municipalité établit ses propres critères spécifiques en vue de déterminer les propriétés qui ont une importance sur le plan local ou régional.

Grâce à ce travail intensif et approfondi, à compter du mois de mai 2006 , on comptait au total 265 propriétés inscrites au registre provincial. Dans la région de Pointe-de-l'Église, on trouve, par exemple, l'église Sainte-Marie, inscrite au registre en janvier 2001, et la maison d'Isaac LeBlanc, inscrite au registre en septembre 1997. L'église Sainte-Marie a été retenue en raison de son architecture de type néo-roman français et parce qu'elle est considérée comme étant le plus haut édifice en bois de l'Amérique du Nord. Elle a également été retenue en raison de sa valeur symbolique importante pour la communauté acadienne de Pointe-del'Église et au-delà. La maison d'Isaac LeBlanc, quant à elle, a été retenue en raison de ses liens avec Hilaire LeBlanc, descendant direct du premier colon acadien établi à Pointe-de-l'Église, de son importance sur le plan culturel et du fait qu'elle constitue un bon exemple du style architectural spécifique de la Nouvelle-Écosse.

Plus de 1000 propriétés ont été inscrites dans des registres municipaux en Nouvelle-Écosse. Cinquante et une des cinquante-six unités municipales de la Nouvelle-Écosse ont adopté des arrêtés en matière de patrimoine et la plupart ont mis en place un comité consultatif sur le patrimoine chargé de l'inscription d'édifices au Registre des biens patrimoniaux de la municipalité. Le district de Clare a bel et bien adopté un arrêté municipal lui permettant d'inscrire des propriétés au Registre des biens patrimoniaux de la municipalité et d'en assurer la protection, et, à l'heure actuelle, ce registre comprend une seule inscription.

La Nouvelle-Écosse compte cinq arrondissements de préservation du patrimoine qui ont été établis : le «Collins Heritage Conservation District » à Yarmouth, le «Old Town Lunenburg Heritage Conservation District » et trois arrondissements résidentiels dans la ville de Truro. Les villes de Truro, de Yarmouth et de Sydney sont toutes en train de se préparer à établir d'autres arrondissements.

Il est possible de retirer un bien patrimonial du registre provincial, lorsque l'édifice a été détruit ou endommagé pour une raison quelconque ou lorsque, de l'avis du ministre, il n'est plus approprié d'inclure la propriété dans le Registre des biens patrimoniaux de la province. Il est également possible de retirer un bien patrimonial d'un registre municipal, pour les mêmes raisons, du moment que cela se fait avec l'approbation du conseil municipal. 
Comme on l'a dit plus haut, le PBP fournit une aide financière par l'intermédiaire de subventions. Les propriétés municipales ont elles aussi accès à une aide financière du même type. La loi sur les biens patrimoniaux attribue au ministre le pouvoir d'accorder aux propriétaires de biens patrimoniaux protégés une aide financière. Les subventions du PBP, qui dispose à l'heure actuelle d'un budget annuel de 50000 dollars, peuvent être décernées pour trois types d'aide : travaux de préservation, conseils concernant la préservation et programme de déductions fiscales provinciales. Certaines administrations municipales, comme la municipalité régionale d'Halifax et la ville d'Annapolis-Royal, accordent elles aussi une aide financière aux propriétaires des biens patrimoniaux inscrits au registre.

\section{Les lois sur les endroits spéciaux et sur les cimetières}

Les ressources du patrimoine provincial de la Nouvelle-Écosse sont également protégées par deux autres lois gérées par la Division du patrimoine du ministère du Tourisme, de la Culture et du Patrimoine : la loi sur les endroits spéciaux (Special Places Act) et la loi sur la protection des cimetières (Cemeteries Protection $A c t$ ). La première a été adoptée par le gouvernement de la Nouvelle-Écosse en 1980. Cette loi favorise l'étude, la protection et la gestion des sites considérés comme des composantes importantes du patrimoine naturel ou humain de la Nouvelle-Écosse et plus particulièrement des sites qui ont de l'importance sur le plan archéologique, historique, paléontologique ou écologique. La loi permet au ministre qui en est responsable de désigner de tels sites comme étant des sites protégés. Les sites protégés peuvent être des propriétés privées ou publiques. L'un des éléments clefs de l'étude et de la gestion de l'ensemble des ressources archéologiques, historiques et paléontologiques est le système de permis pour les biens patrimoniaux. Avec ce système, il est nécessaire de disposer d'un permis pour pouvoir entreprendre toute activité d'exploration ou d'excavation.

La loi la plus récente concernant les ressources patrimoniales provinciales est la loi sur la protection des cimetières, qui date de 1998. Il s'agit d'une loi qui protège non seulement les dépouilles humaines, mais également toute marque, tout monument ou toute structure associée se trouvant sur un terrain protégé par la loi. Les lieux de sépulture représentent une ressource patrimoniale importante en Nouvelle-Écosse, parce qu'ils ont valeur d'archives et d'œuvres d'art pour des communautés qui ont parfois disparu depuis. Lorsqu'on ne connaît pas le propriétaire d'un lieu d'inhumation, qu'on ne peut pas le trouver ou qu'il n'est pas en mesure d'assurer l'entretien du site, la province peut déclarer que le site est un cimetière abandonné et autoriser alors des tierces parties à 
entreprendre le travail de préservation nécessaire. Toute personne prise en flagrant délit de profanation d'une partie quelconque d'un cimetière est en infraction vis-à-vis de cette loi.

\section{Une initiative fédérale}

Ces trois lois permettent aux Néo-Écossais de mieux comprendre les ressources de leur patrimoine et de trouver moyen de les préserver du mieux possible pour les générations à venir. Les progrès réalisés au cours des dernières décennies en matière de préservation ont débouché sur de nouveaux outils et de nouvelles ressources dont la Nouvelle-Écosse dispose pour partager son patrimoine avec les personnes intéressées, au-delà des frontières de la province elle-même. L'Initiative des endroits historiques (IEH) est un nouveau programme national, auquel participent toutes les provinces et tous les territoires du Canada; la province de la Nouvelle-Écosse en fait partie depuis 2003. Le but de l'IEH est d'assurer la protection, la promotion et la préservation des lieux historiques au Canada, y compris les biens désignés par le gouvernement provincial et par les administrations municipales de la Nouvelle-Écosse, les endroits spéciaux et les cimetières. L'IEH s'efforce de favoriser la mise en place d'une " culture de conservation ", en travaillant en collaboration avec les provinces, les territoires, le gouvernement fédéral et les administrations municipales, non seulement pour créer un catalogue complet de tous les lieux historiques au Canada, mais également pour fournir des conseils utiles en matière de gestion des interventions sur les lieux historiques. Ce qui sous-tend ces développements et joue un rôle fondamental, c'est la nécessité de comprendre et d'expliquer les raisons pour lesquelles les lieux historiques ont de la valeur dans leur communauté. Comprendre l'importance d'un lieu, c'est comprendre les valeurs de l'endroit, qui peuvent être, entre autres, des valeurs d'ordre historique, architectural, local ou scientifique. Ces valeurs sont exprimées dans un énoncé d'importance, qui devient un outil important et essentiel pour les communautés et les administrations municipales lorsqu'elles cherchent à prendre des décisions en connaissance de cause sur les changements et les modifications à apporter à un lieu historique. Pour en apprendre davantage sur les lieux historiques de la Nouvelle-Écosse, on peut accéder au Registre des endroits historiques de la Nouvelle-Écosse, qui est une base de données en ligne, contenant des photographies, des descriptions, des notes historiques et des indications aidant à localiser 
les édifices et les lieux historiques dûment enregistrés. Cet outil de vaste ampleur est accessible en ligne ${ }^{1}$.

Il est clair que, au fil des ans, le mouvement de conservation du patrimoine a pris de l'envergure et a évolué. Au cours des dernières années, notre définition du " patrimoine " s'est élargie : à la liste des anciennes églises et grandes demeures qui ont été préservées sont venus s'ajouter des édifices d'exploitation agricole, des paysages urbains, des structures industrielles, des arrondissements historiques et des « paysages culturels ». Nous tenons aussi désormais compte d'un éventail plus varié de récits de connaissances dont ces biens patrimoniaux sont le reflet; autrement dit, notre compréhension de l'histoire de la Nouvelle-Écosse est plus riche et plus variée. Le fait que la Nouvelle-Écosse compte un vaste éventail de nationalités, d'appartenances ethniques et de religions fait de la province une destination populaire chez les touristes et une région enviée pour sa qualité de vie.

Avec cette définition élargie du patrimoine, nous avons également adopté une conception élargie de la gestion de ces biens patrimoniaux et nous comprenons désormais qu'il ne s'agit pas seulement de protéger le patrimoine, mais aussi de gérer les changements, qui sont inévitables. C'est une responsabilité que se partagent de nombreux intervenants, y compris les gouvernements, les groupes de revendication et, tout particulièrement, les propriétaires des biens patrimoniaux. Chaque groupe a un rôle unique et crucial à jouer, mais ce sont, en dernière analyse, les milliers de Néo-Écossais et de Néo-Écossaises, qui sont propriétaires des biens patrimoniaux - inscrits au registre ou non - et qui en prennent soin, qui garantissent que ce patrimoine sera légué sous une forme intacte aux générations à venir de la Nouvelle-Écosse. La Division du patrimoine du ministère du Tourisme, de la Culture et du Patrimoine de la Nouvelle-Écosse espère, par cet exposé, que les participants au colloque en apprendront davantage et se découvriront l'envie d'explorer de façon plus approfondie le patrimoine de leur propre communauté. Les outils, la technologie et l'esprit nécessaires pour prendre en charge ce patrimoine et le partager avec le reste de la province et au-delà sont en place; il s'agit maintenant de profiter des possibilités qui se présentent à nous. Les membres du personnel des programmes mentionnés ci-dessus sont tout à fait prêts à aider les personnes qui auraient des questions au sujet de leur travail.

1. Voir le site 〈www.nshistoricplaces.ca〉. 